\title{
Transcriptomic analysis of Streptococcus agalactiae periprosthetic joint infection
}

\author{
Hye-Kyung Cho ${ }^{1}$, Thao Masters ${ }^{1}$, Kerryl Greenwood-Quaintance ${ }^{1}$, Stephen Johnson ${ }^{1}$, \\ Patricio Jeraldo ${ }^{1}$, Nicholas Chia ${ }^{2}$, Meng Pu${ }^{1}$, Matthew P. Abdel ${ }^{1}$, and Robin Patel ${ }^{1}$
}

\author{
${ }^{1}$ Mayo Clinic \\ ${ }^{2}$ Mayo Clinic Minnesota
}

November 27, 2021

\begin{abstract}
Although Streptococcus agalactiae periprosthetic joint infection (PJI) is not as prevalent as staphylococcal PJI, invasive S. agalactiae infection has recently increased in incidence. Here, RNA-Seq was used to perform transcriptomic analysis of S. agalactiae PJI using fluid derived from sonication of explanted arthroplasties of subjects with S. agalactiae PJI, with results compared to those of S. agalactiae strain NEM316 grown in vitro. 227 genes with outlier expression were found (164 upregulated and 63 down-regulated) between PJI sonicate fluid and in vitro conditions. Functional enrichment analysis showed genes involved in mobilome and inorganic ion transport and metabolism to be most enriched. Genes involved in nickel, copper, and zinc transport, were upregulated. Among known virulence factors, cyl operon genes, encoding beta-hemolysin/cytolysin, were consistently highly expressed in PJI versus in vitro. The data presented provide insight into S. agalactiae PJI pathogenesis and may be a useful resource for the identification of novel PJI therapeutics or vaccines against invasive S. agalactiae infections.
\end{abstract}

\section{Hosted file}

MB032021100489.pdf available at https://authorea.com/users/448279/articles/547092transcriptomic-analysis-of-streptococcus-agalactiae-periprosthetic-joint-infection 